\title{
A search for cold dust around neutron stars
}

\author{
O. Löhmer ${ }^{1}$, A. Wolszczan ${ }^{1,2,3}$, and R. Wielebinski ${ }^{1}$ \\ 1 Max-Planck-Institut für Radioastronomie, Auf dem Hügel 69, 53121 Bonn, Germany \\ e-mail: loehmer@mpifr-bonn.mpg.de \\ 2 Department of Astronomy and Astrophysics, Pennsylvania State University, University Park, PA 16802, USA \\ 3 Toruń Centre for Astronomy, Nicolaus Copernicus University, Gagarina 11, 87-100 Torun, Poland
}

Received 9 May 2004 / Accepted 22 June 2004

\begin{abstract}
We present observations of nine radio pulsars using the Heinrich-Hertz-Telescope at $\lambda 0.87 \mathrm{~mm}$ and the IRAM $30-\mathrm{m}$ telescope at $\lambda 1.2 \mathrm{~mm}$ in search for a cold dust around these sources. Five of the program pulsars have been observed for the first time at the mm-wavelengths. The results are consistent with the absence of circumpulsar disks that would be massive enough $\left(\geq 0.01 M_{\odot}\right)$ to support planet formation according to the scenarios envisioned for solar-type stars, but they do not exclude lower mass $\left(\leq 10-100 M_{\oplus}\right)$ disks for a wide range of grain sizes. These conclusions confirm the previously published results and, together with the current lack of further detections of pulsar planets, they suggest that planet formation around neutron stars is not a common phenomenon.
\end{abstract}

Key words. stars: circumstellar matter - stars: planetary systems - stars: pulsars: general

\section{Introduction}

The existence of planets around one of the millisecond pulsars, PSR B1257+12 (Wolszczan \& Frail 1992; Wolszczan 994), has raised a possibility that at least some pulsars may, like normal stars, be accompanied by protoplanetary or debris disks. Most theories of planet formation around neutron stars assume a creation of some sort of a protoplanetary disk out of the material supplied by the pulsar's binary companion or, possibly, by the fallback of supernova ejecta (e.g., Podsiadlowski 1993; Phinney \& Hansen 1993). Of course, properties and composition of such disks may be significantly different from those of the disks commonly observed around young normal stars (e.g., Boss 2003). In addition, the initial conditions for planet formation in an expanding disk must depend on its ability to protect itself from high-energy photons generated by early accretion onto the pulsar and from a wind of ultrarelativistic particles that carries most of the pulsar's spindown energy (Phinney \& Hansen 1993; Miller \& Hamilton 2001). Although the spindown luminosities of pulsars are, in principle, more than sufficient to heat the dust grains, the efficiency of this process depends on a degree of coupling of the pulsar wind to dust, which is difficult to predict in absence of a sufficient observational evidence.

Since the discovery of the PSR B $1257+12$ planets, there have been several attempts to detect the hypothetical circumpulsar disks with both the space- and the ground-based telescopes. Upper flux limits have been derived for a number of sources at wavelengths ranging from $10 \mu \mathrm{m}$ to
3 mm (van Buren \& Terebey 1993 (IRAF); Zuckerman 1993 (IRTF); Phillips \& Chandler 1994 (JCMT, OVRO); Foster \& Fischer 1996 (IRTF); Greaves \& Holland 2000 (JCMT); Koch-Miramond et al. 2002 (ISO, IRAF/Scanpi); Lazio \& Fischer 2004 (ISO)). As shown by these authors, the existing infrared and mm-wave flux limits for PSR B1257+12 and other pulsars do rule out massive, $\geq 0.01 M_{\odot}$ disks similar to those thought to give rise to planets around normal stars (Boss 2003). However, these limits do not contradict a possibility that some pulsars may be accompanied by much less massive disks ranging from a fraction of the asteroid belt mass to a few hundred $M_{\oplus}$. In fact, the three PSR B1257+12 planets of a total mass of $\sim 8 M_{\oplus}$ appear to have formed from a protoplanetary disk, as suggested by the observed coplanarity of their orbits (Konacki \& Wolszczan 2003). Along these lines, Miller \& Hamilton (2001) have argued that the lower limit to a disk mass necessary to shield the forming PSR B1257+12 planets against the particle and the photon emission of the pulsar would be around $\sim 10^{28} \mathrm{~g}$. Furthermore, Hansen (2002) has considered PSR B1257+12 planet formation scenarios in which the expanded, planet building part of the disk would require a mass comparable to that of the existing planets.

In this paper, we present observations of nine pulsars with the Heinrich-Hertz-Telescope at $\lambda 0.87 \mathrm{~mm}$ and the IRAM $30-\mathrm{m}$ telescope at $\lambda 1.2 \mathrm{~mm}$. Our measurements were designed to either detect or to set upper limits to an emission from possible cool protoplanetary or debris disks around these objects. Five of the program pulsars have been observed at these wavelengths for the first time. We describe the 
Table 1. Continuum flux limits for nine pulsars at $\lambda 0.87 \mathrm{~mm}$ and $\lambda 1.2 \mathrm{~mm}$.

\begin{tabular}{crccrrrc}
\hline \hline PSR & $\begin{array}{c}P \\
(\mathrm{~ms})\end{array}$ & $\begin{array}{c}\dot{P} \\
\left(10^{-18} \mathrm{~s} \mathrm{~s}^{-1}\right)\end{array}$ & $\begin{array}{c}d \\
(\mathrm{pc})\end{array}$ & $\begin{array}{c}\tau \\
(\mathrm{Myr})\end{array}$ & $\begin{array}{c}S_{0.87} \\
(\mathrm{mJy})\end{array}$ & $\begin{array}{c}S_{1.2} \\
(\mathrm{mJy})\end{array}$ & Remarks \\
\hline $\mathrm{J} 0108-1431 \ldots \ldots$. & 807.6 & 77.0 & 130 & 166 & 14.5 & - & solitary PSR \\
$\mathrm{B} 0950+08 \ldots \ldots .$. & 253.1 & 229.8 & 160 & 17.5 & 14.6 & - & solitary PSR \\
$\mathrm{J} 1012+5307 \ldots \ldots$. & 5.3 & 0.017 & 520 & 4860 & - & 2.7 & MSP-WD binary \\
$\mathrm{J} 1024-0719 \ldots \ldots$. & 5.2 & 0.019 & 350 & 4410 & - & 1.8 & solitary MSP \\
$\mathrm{B} 1257+12 \ldots \ldots .$. & 6.2 & 0.114 & 620 & 863 & 7.6 & 2.0 & MSP, 3 planets \\
$\mathrm{B} 1855+09 \ldots \ldots$. & 5.4 & 0.018 & 700 & 4760 & 12.5 & 2.0 & MSP-WD binary \\
$\mathrm{J} 2124-3358 \ldots \ldots$. & 4.9 & 0.021 & 250 & 3800 & 56.0 & - & solitary MSP \\
$\mathrm{J} 2307+2225 \ldots \ldots$. & 535.8 & 8.7 & 380 & 976 & 12.0 & - & solitary PSR \\
$\mathrm{J} 2322+2057 \ldots \ldots$. & 4.8 & 0.010 & 780 & 7850 & 10.2 & - & solitary MSP \\
\hline
\end{tabular}

Notes.- (1) Pulsar distances, $d$, were derived from the Taylor \& Cordes (1993) model of the Galactic electron density distribution. (2) The characteristic age of a pulsar is defined as $\tau=P / 2 \dot{P}$, where $P$ is the pulsar period and $\dot{P}$ is the period derivative. (3) The flux values, $S_{0.87}$ and $S_{1.2}$, are $2 \sigma$ limits.

instrumental setup and present flux density measurements of the nine sources in Sect. 2. In Sect. 3, these and other results are further discussed in the context of the recent observational and theoretical developments.

\section{Observations and data analysis}

The sample of pulsars selected for our survey consisted of nine nearby sources within the distance limit of $<1 \mathrm{kpc}$. Alongside with the planets pulsar, PSR B1257+12, we have included three other solitary millisecond pulsars (MSPs) on the assumption that they would be the most probable candidates for a detectable dust emission. As binary MSPs must have undergone long periods of accretion from their companions and could be left with a residual circumstellar material suitable for planet formation (Phinney \& Hansen 1993), two MSP-WD (white dwarf) binaries have been also included. Finally, for completeness, we have supplemented our list with the three nearby normal pulsars that would be observable at the telescope sites.

The continuum observations at $\lambda 0.87 \mathrm{~mm}$ were carried out at the Heinrich-Hertz-Telescope (Baars et al. 1999) on Mt. Graham, Arizona, during two observing sessions in January 2001 and December 2002. We used a sensitive 19-channel bolometer array developed by E. Kreysa and collaborators at the Max-Planck-Institut für Radioastronomie, Bonn. The centre frequency of the bolometer was about $345 \mathrm{GHz}$, with a bandwidth $70 \mathrm{GHz}$. The beam size on the sky was $\sim 23^{\prime \prime}$. Using the central channel of the bolometer, we performed standard on-off measurements with the telescope secondary chopping in azimuth by $40^{\prime \prime}$. In order to calculate the atmospheric zenith opacity, we made skydip observations every $45 \mathrm{~min}$. The resulting opacities at the observing frequency varied between 0.3 and 0.9 during the two observing sessions. For calibration purposes we performed mapping and on-off measurements of various planets (mainly Mars, Saturn and Uranus), resulting in conversion factors of 7.7-9.5 mJy per count.

The observations at $\lambda 1.2 \mathrm{~mm}$ were carried out in February and March 2003 at the IRAM 30-m telescope on Pico Veleta (Spain). We used the 117-channel Max-Planck Millimeter
Bolometer (MAMBO-2) array (Kreysa et al. 1999), which had a half-power spectral bandpass from 210 to $290 \mathrm{GHz}$ with an effective frequency of $250 \mathrm{GHz}$. The beam size on the sky was 10.7 arcsec. The sources were observed with a single channel using the standard on-off mode with the telescope secondary chopping in azimuth by $40^{\prime \prime}$ at a rate of $2 \mathrm{~Hz}$. The sky opacity at the observing frequency was monitored with skydips and varied between 0.1 and 0.3 during the seven days of the observing run. For flux calibration we observed a number of calibration sources, resulting in conversion factors of 32-37 counts per mJy and an estimated absolute flux uncertainty of $15 \%$.

The data obtained at the two telescopes were converted into a standard format and analysed using the NIC software package. Correlated noise was subtracted from the reference channel using the weighted average signals from the surrounding channels. Anomalous signals were clipped above the $5 \sigma$ level.

We did not detect dust emission towards any of the pulsars observed. Table 1 lists the $2 \sigma$ flux limits obtained at the two observing frequencies. At $\lambda 0.87 \mathrm{~mm}$ we reached limits of 7-14 mJy for most of the sources, which is slightly more than the limits of the survey by Greaves \& Holland (2000). At $\lambda 1.2 \mathrm{~mm} 2 \sigma$ flux limits of 1.8-2.7 mJy were obtained.

\section{Discussion}

A lack of detections and the derived flux limits at $\lambda 0.87 \mathrm{~mm}$ and $\lambda 1.2 \mathrm{~mm}$ for the nine pulsars discussed in this paper (Table 1) place useful constraints on the existence of cold, dusty disks around these sources. To illustrate the implications of this result for a circumpulsar disk observability in the framework of a simple model, we use the approach of Foster $\&$ Fischer (1996) by assuming that a fraction $f$ of the pulsar spindown luminosity $\dot{E}$ heats a disk of mass $m_{\mathrm{d}}$ consisting of dust grains of size $a$ to a temperature $T$, and calculate the expected infrared flux as a function of disk parameters and pulsar distance following the formalism developed by these authors. Because PSR B1257+12 has planets that have originated in a disk (Konacki \& Wolszczan 2003), it is the best sampled pulsar in the sub- $\mathrm{mm} / \mathrm{mm}$ range, and the published disk mass limits 


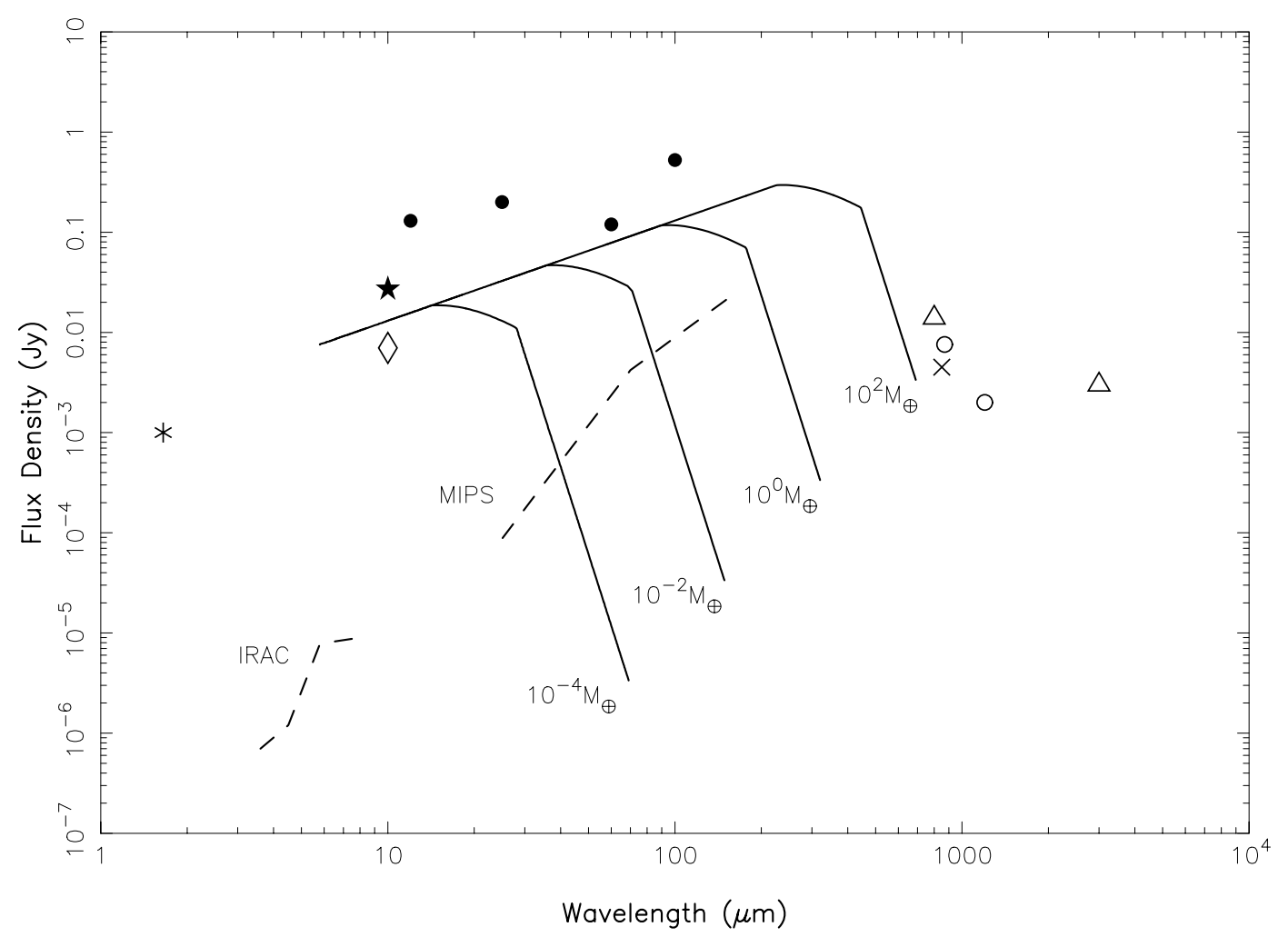

Fig. 1. Observational limits and theoretical models for the emission from dust in the PSR B1257+12 planetary system. Symbols mark the flux limits set by observations discussed in the text (०) and by published measurements with the following instruments and surveys: IRTF $(\star, \diamond)$, IRAS/Scanpi $(\bullet)$, JCMT/OVRO $(\triangle, \times)$, and 2MASS $(*)$. The solid curves trace the loci of a maximum flux for theoretical disk emission models calculated for four disk masses as a function of the grain size ranging from 0.01 to $1000 \mu \mathrm{m}$. Dashed lines correspond to the Spitzer Space Telescope $3 \sigma$ sensitivity limits estimated for the IRAC and the MIPS detectors.

are not dramatically different from one pulsar to another, we can use this object as a representative example to make a general comparison of model calculations with the data.

In Fig. 1, four sets of models for disk masses $m_{\mathrm{d}}=10^{-4}$, $10^{-2}, 10^{0}$, and $10^{2} M_{\oplus}$ are compared with the flux limits for PSR B1257+12 obtained in this work and the ones published elsewhere. To ensure compatibility with other results, we have assumed $k=0.01$ (Foster \& Fischer 1996), $0.01 \mu \mathrm{m} \leq a \leq$ $1000 \mu \mathrm{m}$ (Koch-Miramond et al. 2002), the standard spindown luminosity of the pulsar, $\dot{E}=2 \times 10^{34} \mathrm{erg} \mathrm{s}^{-1}$, and the pulsar distance of 620 pc from the Taylor \& Cordes (1993) model of the Galactic electron density distribution. In the modelling process, for the assumed disk mass and grain size ranges, the grain temperature varied over the range of $8 \mathrm{~K} \leq T \leq 500 \mathrm{~K}$, from the largest to the smallest values of $m_{\mathrm{d}}$ and $a$, respectively. This is practically within the range between the typical temperature of the cold interstellar dust $(10 \mathrm{~K})$ and the sublimation temperature of silicate grains $(1500 \mathrm{~K})$ (e.g., Koch-Miramond et al. 2002).

A comparison of the existing observations with a simple dust model shown in Fig. 1 clearly demonstrates the absence of a disk around PSR B1257+12 with the mass $\geq 0.01 M_{\odot}$ that appears to be necessary to support planet formation around solar-type stars (Boss 2003). This conclusion is also valid for our data on other pulsars, and it is generally consistent with the limits on dusty disk masses obtained from previous observations of a number of pulsars in the $\lambda 10 \mu \mathrm{m}-\lambda 3 \mathrm{~mm}$ range (e.g., Greaves \& Holland 2000; Koch-Miramond et al. 2002). Of course, one could alter this outcome by varying the heating efficiency of the disk by the pulsar wind, or changing the disk model assumptions. For example, an upper limit in excess of $0.01 M_{\odot}$ can be obtained for the mass of a disk around PSR B1257+12 by extrapolating from models of the T Tauri disks (Phillips \& Chandler 1994). Similarly, a dusty disk model used by Jura (2003) to explain an infrared excess in the white dwarf G29-38 can, in principle, be modified to describe the illumination of a disk by nonthermal radiation from a neutron star (M. Jura, personal communication). However, these results cannot be conclusively compared with the ones based on more generic models (e.g., Foster \& Fischer 1996; Koch-Miramond et al. 2002) without a much better understanding of the physics of hypothetical circumpulsar disks.

As stated earlier and evident from Fig. 1, the data do not exclude a possibility that dusty disks ranging from a fraction of the mass of the solar system asteroid belt up to possibly $\sim 100 M_{\oplus}$ may exist around pulsars over a wide range of temperatures and grain sizes. Conditions for the existence of such disks have been recently examined by Miller \& Hamilton (2001), who specifically predict that a leftover material cannot exist around pulsars without planets. This is because such pulsars have evidently not managed to develop protoplanetary disks with a sufficient mass $\left(m_{\mathrm{d}} \geq 10^{28} \mathrm{~g}\right.$ for PSR B1257+12) to protect themselves against evaporation by the pulsar wind and accretion flux that would prevent planet formation. 
On the other hand, pulsars with planets, like PSR B1257+12, could still have observable debris disks left over from the final stages of a protoplanetary disk evolution (e.g., Hansen 2002). In principle, dramatically more sensitive observations of PSR B1257+12 and other pulsars with the Spitzer Space Telescope will have a potential to either reveal such a circumpulsar debris material or to place very meaningful constraints on its existence (Fig. 1).

Our data support a general conclusion drawn from the previous negative results of searches for dust emission from the vicinity of pulsars that planet formation around neutron stars occurs infrequently and that it follows an evolutionary path that must generally be different from the one envisioned for planets around normal stars. This conclusion particularly refers to possible sources of a circumpulsar matter, as well as to the problem of a disk formation and retention around a neutron star (Podsiadlowski 1993; Phinney \& Hansen 1993; Miller \& Hamilton 2001). It is also consistent with a crude statistic of $<10 \%$ derived from the fact that PSR B1257+12, one out of the nine known solitary millisecond pulsars, has planets around it (Konacki \& Wolszczan 2003). Similarly, it is not surprising that no massive dusty disks have been found around relatively few normal, young pulsars observed so far, as the formation and survival of planets around such objects may be particularly difficult (Thorsett \& Dewey 1993).

In the absence of new detections of planets around millisecond pulsars (the Jupiter-mass planet in the M 4 globular cluster, recently confirmed by Sigurdsson et al. (2003), was probably created around a normal star) further searches for dust around these objects, covering all the physically plausible parameter space at a possibly high sensitivity level, is a logical way to meaningfully constrain the models of a creation and evolution of pulsar protoplanetary and debris disks. Given the existing correlation between stellar metallicity and the frequency of planets around normal stars (Santos et al. 2003; Fischer et al. 2004), it is likely that the protoplanetary disks around pulsars, possibly made of a highly evolved stellar material, do not have to be very massive to support an efficient planet formation (see also Lazio \& Fischer 2004). Furthermore, one would also have to include a distinct possibility that the "dusty plasma" environment of a circumpulsar disk (e.g., Mendis \& Rosenberg 1994), driven by the relativistic pulsar wind and the magnetic fields carried with it, cannot be satisfactorily approximated by the standard protoplanetary or debris disk models based on theories and observations of the formation regions of solar-type stars (e.g., Boss 2003). In the near future, the Spitzer Space Telescope, with its factor of $\sim 10^{2}-10^{4}$ improvement in sensitivity compared to the instruments previously used to search for dust emission from around pulsars (Fig. 1), is an obvious choice for further exploration of the physics of neutron star planetary systems.

Acknowledgements. We are very grateful to the staff at the HHT and the 30-m telescope for their excellent support. We thank M. Dumke and M. Thierbach for their help during the observations. A.W.'s research was supported by the Alexander von Humboldt Foundation, the NASA grant NAG5-13620, and by the NSF under Grant No. PHY99-07949.

\section{References}

Baars, J. W. M., Martin, R. N., Mangum, J. G., McMullin, J. P., \& Peters, W. L. 1999, PASP, 111, 627

Boss, A. P. 2003, in Scientific Frontiers in Research on Extrasolar Planets, ASP Conf. Ser., 294, 269

Fischer, D., Valenti, J. A., \& Marcy, G. 2004, Stars as Suns: Activity, Evolution and Planets, IAU Symp., 219, in press

Foster, R. S., \& Fischer, J. 1996, ApJ, 460, 902

Greaves, J. S., \& Holland, W. S. 2000, MNRAS, 316, L21

Hansen, B. M. S. 2002, in Stellar Collisions, Mergers and their Consequences, ed. M. M. Shara, ASP Conf. Ser., 263, 221

Jura, M. 2003, ApJ, 584, L91

Koch-Miramond, L., Haas, M., Pantin, E., et al. 2002, A\&A, 387, 233

Konacki, M., \& Wolszczan, A. 2003, ApJ, 591, L147

Kreysa, E., Gemünd, H.-P., Gromke, J., et al. 1999, Infr. Phys. Technol., 40, 191

Lazio, T. J. W., \& Fischer, J. 2004 [arXiv: astro-ph/0405344]

Mendis, D. A., \& Rosenberg, M. 1994, ARA\&A, 32, 419

Miller, M. C., \& Hamilton, D. P. 2001, ApJ, 550, 863

Phillips, J. A., \& Chandler, C. J. 1994, ApJ, 420, L83

Phinney, E. S., \& Hansen, B. M. S. 1993, in Planets around Pulsars, ed. J. A. Phillips, S. E. Thorsett, \& S. R. Kulkarni, ASP Conf. Ser., 36,371

Podsiadlowski, P. 1993, in Planets around Pulsars, ed. J. A. Phillips, S. E. Thorsett, \& S. R. Kulkarni, ASP Conf. Ser., 36, 149

Santos, N. C., Israelian, G., Mayor, M., Rebolo, R., \& Udry, S. 2003, A\&A, 398, 363

Sigurdsson, S., Richer, H. B., Hansen, B. M., Stairs, I. H., \& Thorsett, S. E. 2003, Science, 301, 193

Taylor, J. H., \& Cordes, J. M. 1993, ApJ, 411, 674

Thorsett, S. E., \& Dewey, R. J. 1993, ApJ, 419, L65

van Buren, D., \& Terebey, S. 1993, in Planets around Pulsars, ed. J. A. Phillips, S. E. Thorsett, \& S. R. Kulkarni, ASP Conf. Ser., 36, 327 Wolszczan, A. 1994, Science, 264, 538

Wolszczan, A., \& Frail, D. A. 1992, Nature, 355, 145

Zuckerman, B. 1993, in Planets around Pulsars, ed. J. A. Phillips, S. E. Thorsett, \& S. R. Kulkarni, ASP Conf. Ser., 36, 303 